Rapid Communications

\title{
Live Single-cell Metabolomics of Tryptophan and Histidine Metabolites in a Rat Basophil Leukemia Cell
}

\author{
Hajime Mizuno, Naohiro Tsuyama, Sachiko Date, Takanori Harada, and Tsutomu Masujima ${ }^{\dagger}$ \\ Graduate School of Biomedical Sciences, Hiroshima University, \\ 1-2-3 Kasumi, Minami, Hiroshima 734-8553, Japan
}

\begin{abstract}
A direct and rapid metabolic analysis of a live single cell was performed by live single-cell video-mass spectrometry. The contents of the cytoplasm and a granule were sucked into a nano-electrospray ionization (nano-ESI) tip, and were directly introduced into a Q-TOF mass spectrometer by nano-spray after the addition of an ionization solvent. The metabolic pathways and the locations of tryptophan and histidine metabolites were traced by this method for a cultured rat basophil leukemia cell line (RBL-2H3). The $t$-values of detected peaks by a $t$-test between the different location, $e$.g. cytoplasm and a granule, revealed the molecular localization of each MS peak. A direct and quick metabolomic analysis of a living cell under simultaneous video-microscopic observations was innovated.
\end{abstract}

(Received October 17, 2008; Accepted November 17, 2008; Published December 10, 2008)

We have developed a method involving mass spectrometry (MS) that can directly detect various small molecules in a visualized reacting single-cell, called "video-mass spectrometry"1-3 in which a pico liter level of single-cell content was sucked into a nanospray tip under a microscope and the trapped sample is directly introduced into a mass spectrometer by nano-spray ionization. ${ }^{1,2}$ Since the main aim of this new method is to disclose the molecular mechanism of a cell in a direct and rapid way, the analysis of molecular metabolites and those metabolic pathways, i.e. metabolomics, is one of the most important applications.

Metabolomics is a growing analytical field by using MS.4-7 However, as in the case of current bio-analyses, many cells are used for each analysis as a sample at a time, and the results are more or less averaged. We know that the cell response is not the same even in the same circumstances. ${ }^{3}$ Furthermore, the intracellular locality of each metabolite seems to be hard to be discriminated by the averaged method. Thus, the currently proposed metabolic pathways still lack information concerning cell individuality and site specificity in a cell. We found that video-mass spectrometry for a single cell could discover the molecular metabolism in each cell together with providing information about the molecular locality.

Adherent cell line, rat basophil leukemia cell line (RBL-2H3), which has sub-cellular organs, e.g. granules, was cultured in Dulbecco's modified Eagle minimal essential medium, ${ }^{2}$ and the cells were observed by a CCD video camera mounted on an inverted microscope (OLYMPUS, IX-70). The cell contents, granule or cytoplasm of a target RBL-2H3 cell was sucked into a gold coated glass capillary nano-electrospray tip (Humanix, Cellomics-Tip, Japan) by using a micro-manipulator with a tube connected syringe.

For nano-ESI mass spectrometric analysis, the ionization solvent of acetonitrile containing $0.5 \%$ formic acid was added to the sucked sample solution in the capillary of the nanospray tip.

† To whom correspondence should be addressed.

E-mail: tsutomu@hiroshima-u.ac.jp
A mass spectrometric analysis was performed by a Q-TOF mass spectrometer (Applied Biosystems, QSTAR-XL, USA) equipped with a nano-ESI ion source. The spray voltage for nano-ESI was aproximately $1000 \mathrm{~V}$. The spectrometer was calibrated at the beginning of a measurement, and then every $1 \mathrm{~h}$ by dopamine; $m / z 154.0868[\mathrm{M}+\mathrm{H}]^{+}$peak and angiotensin $\mathrm{I} ; \mathrm{m} / \mathrm{z}$ $432.8998[\mathrm{M}+3 \mathrm{H}]^{3+}$ peak. The intensities of the MS peaks were normalized by the intensity of a solvent peak at $\mathrm{m} / \mathrm{z}, 381.26$. MS/MS analysis was performed with the collision energy being from 10 to $30 \mathrm{eV}$ by using nitrogen gas as the collision source. The obtained spectra were analyzed by Markerview (Applied Biosystems, USA) software for a $t$-test analysis. For molecular identification by MS/MS analysis, we referred to the MassBank.jp (http://www.massbank.jp/index.html).

Since the mast cell secretes histamin and serotonin upon stimulation, we focused to apply our live single-cell MS method to analyze the histidine and tryptophan metabolic pathways in a single cell based on information of these local distributions. As shown in Fig. 1, about 700 peaks of the mass spectra were measured in the positive ion detection mode from cytoplasm and a granule in a single RBL-2H3 cell, which is the model cell-line of mast cells; 5 peaks of histidine and tryptophan metabolites were detected at $\mathrm{m} / \mathrm{z}, 112,156,177,205$ and 221.

A statistic analysis of a $t$-test was performed for these 5 peaks $(n=5)$ to clarify the sub-cellular localization between the granules and the cytoplasm. The $t$-values, indicated under the $\mathrm{m} / \mathrm{z}$ values in Fig. 1, show site-dependent distributions of the peaks. In this analysis, the $t$-value of $+100 \%$ shows that the peak is found in a granule specifically, while a $t$-value of $-100 \%$ shows that in cytoplasm specifically. When the $t$-value of a peak is close to zero, the molecule of the peak is distributed in both sites evenly.

The peak at $\mathrm{m} / \mathrm{z}, 112$ was identified as being histamine by a MS/MS analysis (Fig. 2(b)) and the $t$-value of $99.5 \%$ shows that the histamine located specifically in the granules. The specific peak in the granule at $\mathrm{m} / \mathrm{z} 156$, whose $t$-value is $95.2 \%$, was determined as histidine by MS/MS analysis (Fig. 2(a)). According to a metabolic map, ${ }^{8}$ histamine is the metabolite of 

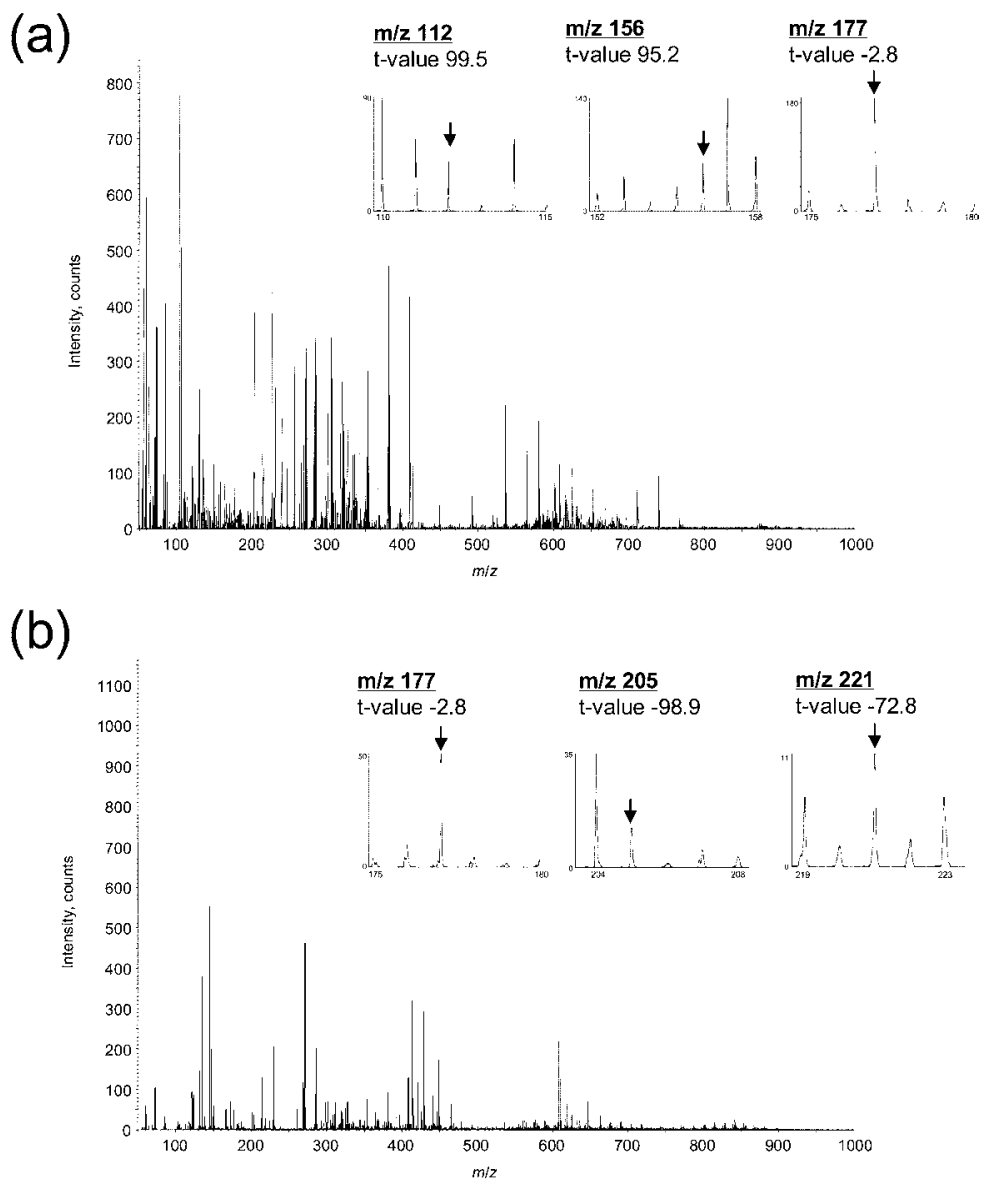

Fig. 1 Nano-ESI-TOF mass spectra of a granule (a) and cytoplasm (b) of a single RBL-2H3 cell. The peaks of related metabolites of histidine and tryptophan are shown by arrowheads in magnified spectra. The mass numbers and $t$-values are also shown in these spectra.

histidine by L-histidine decarboxylase (HDC). Although there is a report that HDC locates not only in the granules, but in the cytoplasm, ${ }^{9}$ the histamine seems to be metabolically biosynthesized in granules from histidine, which mainly exists in the granules in this type of cell line.

On the other hand, the peak at $\mathrm{m} / \mathrm{z} 177$ was found not only in granules but also in the cytoplasm. By MS/MS analyses, both peaks at $\mathrm{m} / \mathrm{z}, 177$ were identified as serotonin as shown in Fig. 2(d). For serotonin peak $(\mathrm{m} / \mathrm{z}, 177.12)$, there is inevitable overlapping of solvent peak at $\mathrm{m} / \mathrm{z}$ 177.17. The solvent peak can not be rejected on MS/MS mode by low filtering ability of a quadrupole MS filter. Thus, the MS/MS spectra of serotonin (d) was also compared with the standard spectrum at low concentration and a characteristic peak at $\mathrm{m} / \mathrm{z} 160.1$ was found which was detected only for serotonin standard sample but not for only the solvent. The $t$-value of $-2.8 \%$ shows that serotonin almost evenly exists not only in the granules but in the cytoplasm of a cell.

A metabolic map ${ }^{10}$ shows that serotonin is the final metabolite of tryptophan by 5-monooxygenase (to produce 5-hydroxytryptophan as the intermediate metabolite $)^{11}$ and aromatic-L-amino acid decarboxylase.

The detected peaks at $\mathrm{m} / \mathrm{z} 205$ and 221 were identified as being from tryptophan and 5-OH tryptophan by MS/MS, as shown in Figs. 2(c) and 2(e), respectively. Since the $t$-values of the peaks of tryptophan and 5-OH tryptophan are -98.9 and $-72.8 \%$, respectively, two substrates to produce serotonin exist in cytoplasm. Thus, it is considered that serotonin is transported into the granules and stored after biosynthesis in the cytoplasm. These results propose the metabolic and transport pathways in a cell, as shown in Fig. 3. The difference in the localization of related metabolites of histamine and serotonin in a single cell reflects the difference in each biosynthetic site and the process in a cell.

The video-mass spectrometry of a single cell has enabled us to analyze the metabolic pathways and the processing mechanism of small molecules of interest, and it will be used in various metabolomics in a single cell. As far as we know, it is the first success of "live single-cell metabolomics" with information about sub-cellular locality and their local metabolic pathways.

\section{Acknowledgements}

We thank the Research Center for Molecular Medicine and the Analysis Center for Life Sciences, Hiroshima University for use of their instruments. This work was supported by Grants-in-Aid for Basic Sciences from the Ministry of Education, Science, Sports and Culture, Japan (No. 20229003 (T. M.), 19590041 (N. T.), and 20790038 (H. M.)).

\section{References}

1. N. Tsuyama, H. Mizuno, E. Tokunaga, and T. Masujima, Anal. Sci., 2008, 24, 559. 
(a)

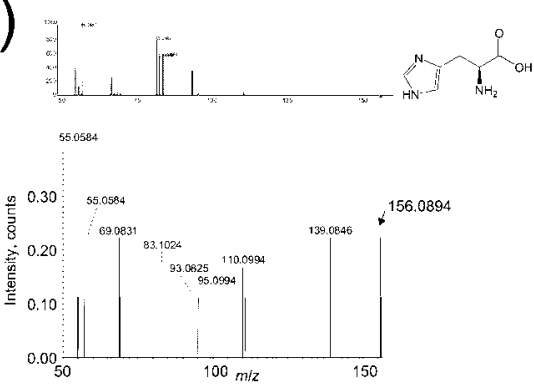

(c)

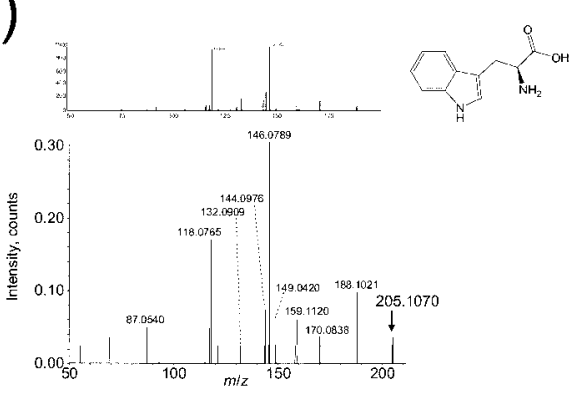

(e)

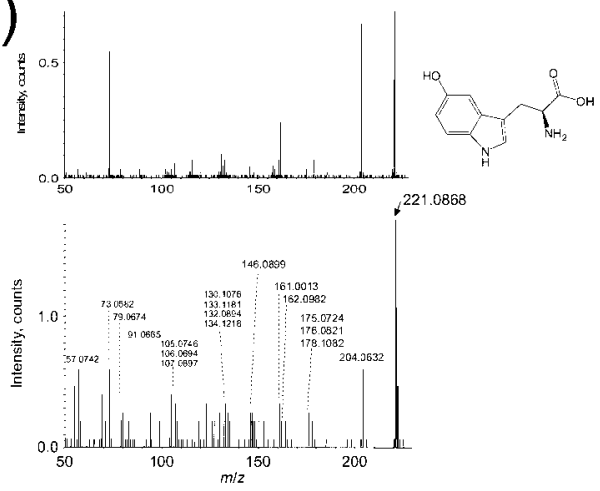

(b)

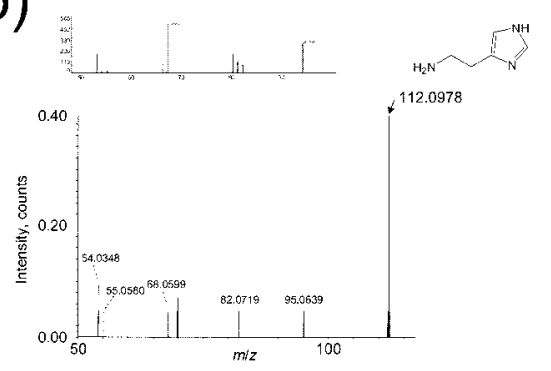

(d)
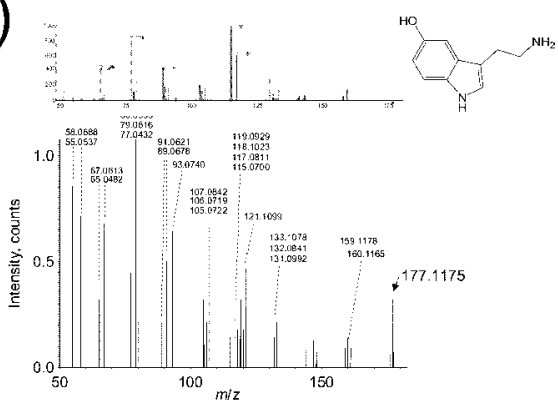

Fig. 2 MS/MS spectra of histidine and tryptophan metabolites. Ion peaks at $m / z, 156$ (a), 112 (b), 205 (c), 177 (d), and 221 (e) were identified to be histidine, histamine, tryptophan, serotonin, and 5-hydroxytryptophan, respectively. The upper spectra of (a) - (d) are reference MS/MS spectra from MassBank, and that of (e) is a 5hydroxytryptophan standard MS/MS spectrum. Identified fragment peaks are indicated by $m / z$ values.

\section{RBL-2H3 cell}

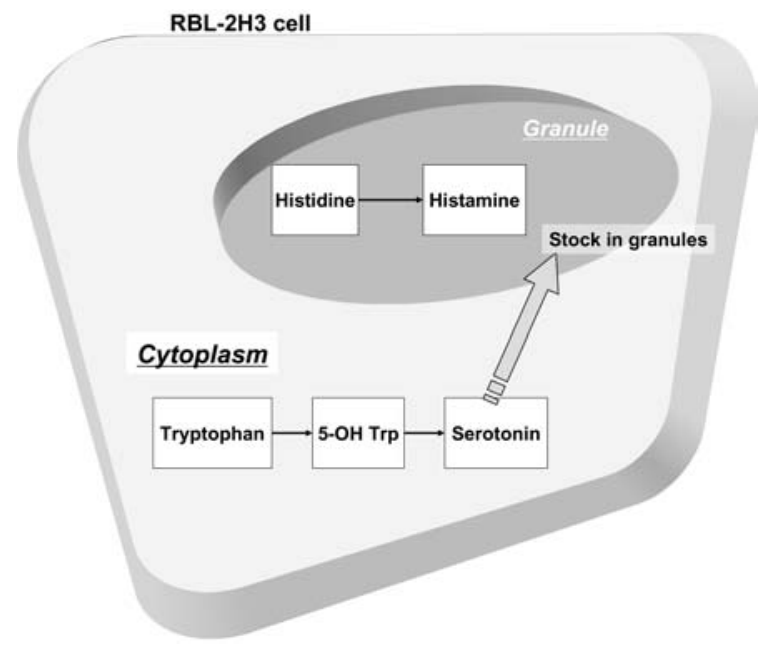

Fig. 3 Proposed schema of the metabolic pathways and locations of tryptophan and histidine metabolites in a single RBL-2H3 cell.
2. H. Mizuno, N. Tsuyama, T. Harada, and T. Masujima, J. Mass. Spectrom., 2008, 43, 1692.

3. T. Masujima, Anal. Chim. Acta, 1999, 400, 33.

4. S. G. Oliver, M. K. Winson, D. B. Kell, and F. Baganz, Trends Biotechnol., 1998, 16, 373.

5. O. Fiehn, J. Kopka, P. Dormann, T. Altmann, R. N. Trethewey, and L. Willmitzer, Nat. Biotechnol., 2000, 18, 1157.

6. R. Goodacre, S. Vaidyanathan, W. B. Dunn, G. G. Harrigan, and D. B. Kell, Trends Biotechnol., 2004, 22, 245.

7. J. L. Griffin and J. P. Shockcor, Nat. Rev. Cancer, 2004, 4, 551.

8. http://www.genome.ad.jp/dbget-bin/show_pathway?ko00340.

9. S. Tanaka, K. Nemoto, E. Yamamura, and A. Ichikawa, J. Biol. Chem., 1998, 273, 8177.

10. http://www.genome.ad.jp/dbget-bin/show_pathway?ko00380.

11. H. Hasegawa, K. Oguro, Y. Naito, and A. Ichiyama, Eur. J. Biochem., 1999, 261, 734. 